\title{
Cloning and Characterization of NAD-dependent Deacetylase Sirtuin 2 Homolog from the Silkworm, Bombyx mori.
}

\author{
Yijia Li \\ Institute of Life Sciences, Jiangsu University \\ 301 Xuefu Road, Zhenjiang 212013, China \\ E-mail: yijia_li@yahoo.com.cn \\ Keping Chen (Corresponding author) \\ Institute of Life Sciences, Jiangsu University \\ 301 Xuefu Road, Zhenjiang 212013, China \\ E-mail: kpchen@ujs.edu.cn
}

Qin Yao

Institute of Life Sciences, Jiangsu University

301 Xuefu Road, Zhenjiang 212013, China

E-mail: yaoqin@ujs.edu.cn

Lu Gao

Institute of Life Sciences, Jiangsu University

301 Xuefu Road, Zhenjiang 212013, China

E-mail: gaolu197657@yahoo.com.cn

Jun Li

Institute of Life Sciences, Jiangsu University

301 Xuefu Road, Zhenjiang 212013, China

E-mail: lij_023@163.com

Lin Wang

Beijing Entry-Exit Inspection and Quarantine Bureau

No. 6 Tianshuiyuan Street, Chaoyang District, Beijing 100026, China

The research is financed by the National "863" project of China. NO. 2008 AA10Z145 and the National Basic Research Program of China. No.2005CB121000. (Sponsoring information)

\section{Abstract}

Sirtuin2 (Sirt2) is a kind of $\mathrm{NAD}^{+}$-dependent deacetylases ranging from bacteria to human and play an important role in many biological processes especially in lifespan. We performed genome analysis and protein prediction of Sirt2 of $B$. mori (BmSirt2). The cDNA sequence of BmSirt2 contains an ORF of $1164 \mathrm{bp}$ encoding 387 amino acid residues with a 
predicted molecular mass and isoelectric point of $43.37 \mathrm{kDa}$ and 5.02, respectively. This protein shows high degrees of identity with other species. Phylogenetic relationship analysis showed that the BmSirt2 protein was in the same subgroup as the Sirt2 from invertebrate animals. RT-PCR analysis of gene expression in multiple tissues showed that Sirt2 gene was widely expressed in B. mori. BmSirt2 was successfully expressed in E. coli with a molecular mass of $48.0 \mathrm{kDa}$. The identification of the recombinant protein by MALDI-TOF-MS and western blotting showed this fusion protein was the correct one.

Keywords: Bombyx mori, Sirtuin2, Bioinformatics, RT-PCR, Prokaryotic expression, Mass spectrographic

\section{Introduction}

Sirt2 enzymes, also known as sirtuins, or silence information regulator (Sir), comprise an ancient family of $\mathrm{NAD}^{+}$-dependent deacetylases conserved from bacteria to human and play a role in a wide variety of important biological processes, including development, heterochromatin formation, transcriptional silencing, DNA recombination and repair, genome stability, apoptosis, axonal protection, fat mobilization, metabolic regulation, and longevity (Avalos, Bever, \& Wolberger, 2005, p.855; Hoff et al., 2006, p.1231). Sirtuins link aging, cancer, and diet and thus are potential molecular targets for the development of pharmaceuticals to treat human metabolic and neurological diseases and malignancy (Schuetz et al., 2007, p.377).

Sirt2 enzymes comprise a universally conserved family of $\mathrm{NAD}^{+}$-dependent deacetylases, which of the reaction can be thought of as occurring by a two-step mechanism. In the first step, two substrates, an acetylated protein and NAD, interact with the active site of the enzyme. The first reaction is reversible in the presence of excess nicotinamide:

$\mathrm{NAD}+$ enzyme + acetyl-lysine $\Longrightarrow$ nicotinamide + enzyme $\sim$ ADP-ribose-acetyl-lysine.

In the second step the high-energy ADP-ribose intermediate reacts with the acetyl group on the e amino group of the lysine:

Enzyme $\sim$ ADP-ribose-acetyl-lysine $\Longrightarrow$ lysine + enzyme + acetyl-ADP-ribose.

(Landry \& Sternglanz, 2003, p.34).

Sirtuins was first found in $S$. accharomyces cereviae and ranging from bacteria to humans. The hallmark of the family is a domain of approximately 260 amino acids that has a high degree of sequence similarity in all sirtuins. The family is divided into five classes (I-IV and U) on the basis of a phylogenetic analysis of 60 sirtuins from a wide array of organisms (Frye, 2000, p.793). Class I and class IV are further divided into three and two subgroups, respectively. The U-class sirtuins are found only in Gram-positive bacteria. The $S$. cerevisiae genome encodes five sirtuins, Sir $2 p$ and four additional proteins termed 'homologs of sir two' (Hstlp-Hst4p) (North \& Verdin, 2004, p.224.1).The human genome encodes seven sirtuins, with representatives from classes I-IV (Frye, 2000, p.793).

In this paper, Sirt2 of Bombyx mori (BmSirt2) was cloned and analyzed with bioinformatics tools, including its genomic organization and the deduced amino acid sequence. The gene was confirmed by RT-PCR and prokaryotic expression. The deduced protein sequence was compared with that of some homologous protein from other species.

\section{Materials and methods}

\subsection{Animals and RNA extraction}

The silkworm, B. mori used in this study, was reared routinely on fresh mulberry leaves at $25^{\circ} \mathrm{C}$. Epidermis, midgut, fat body, silk gland, hemolymph, nerve knot, testis and ovary were dissected and washed in PBS (137 mM NaCl, $2.7 \mathrm{mM}$

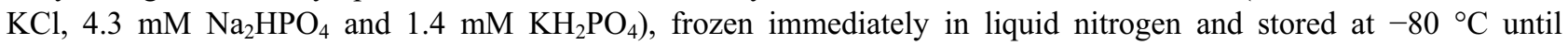
extraction of RNA. Total RNA was isolated from $30 \mathrm{mg}$ each of $B$. mori larvae tissues using the RNeasy® Mini Kit (Qiagen, Valencia, CA, USA). The extracted RNA was treated with RNase-free DNase (Promega, Madison, WI, USA), then was used to synthesize first strand cDNA using the SuperScript II ${ }^{\mathrm{TM}}$ reverse transcriptase (Promega) following the manufacturer's instructions (Wang et al., 2008, p.177).

\subsection{Genome analysis for BmSirt2}

A putative cDNA of BmSirt2 (AB194684) was obtained by the BLAST program using XP_001350011 from Plasmodium falciparum (Chakrabarty et al., 2008, p.140). The DNAstar software was used to find the open reading frame (ORF). TATA box and poly-A signal was predicted by Primer premier software. SIM4 (http://pbil.univ-lyon1.fr/sim4.php) was used to align the cDNA sequence with the genomic sequences to search potential introns. SilkMap of BGI (http://silkworm.genomics.org.cn/) was used to find the location of BmSirt2 on Chromosome.

\subsection{Protein prediction and analysis}

The deduced amino acid sequence was analyzed by the Expert Protein Analysis System (http://www.expasy.org/). Homologues were explored by BLASTP searching tool in the updated GenBank/EMBL databases. Multiple sequence 
alignment was performed by Clustal W (http://www.ebi.ac.uk/clustalw/) and edited using GeneDoc software. Phylogenetic relationships were assessed and trees generated by the neighbour-joining method using MEGA 3.1 (http://www.megasoftware.net).

\subsection{Cloning, sequencing and expression of BmSirt2}

An ORF region of BmSirt2 was amplified by PCR using the forward primer 5'-AGGATCCATGTCGGCAAATTCGCCT-3' and the reverse primer 5'-ACTCGAGTCATTCCGAGGCACTGGG-3' containing $B a m \mathrm{HI}$ and $\mathrm{XhoI}$ restriction sites (underlined) respectively. Actin A3 was amplified using the forward primer 5'-GCGCGGCTACTCGTTCACTACC-3' and the reverse primer 5'-GGATGTCCACGTCGCACTTCA-3'. PCR reaction was carried out for 35 amplification cycles $\left(94^{\circ} \mathrm{C} / 30 \mathrm{sec}, 62^{\circ} \mathrm{C} / 30 \mathrm{sec}, 72^{\circ} \mathrm{C} / 1 \mathrm{~min}\right)$.

The PCR product was ligated into pMD19-T (TaKaRa, Dalian) vector using T4 DNA ligase (TaKaRa, Dalian) and then transformed into E. coli (TG1 strain). After digestion with BamHI and XhoI from the plasmid pMD19-T/BmSirt2, the purified DNA fragment was ligated into expression vector pET-30a (Novagen) and introduced into E. coli BL21 (DE3) by transformation. Sequencing was performed in Shanghai Sangon Bio-technology Corporation using the plasmid pMD19-T/BmSirt2.

The expression of recombinant BmSirt 2 tagged with histidine residues was induced overnight at $28^{\circ} \mathrm{C}$ by the addition of isopropyl- $\beta$-D-thiogalactopyranoside (IPTG) to a final concentration of $1.0 \mathrm{mM}$ when the optical density of the culture at $600 \mathrm{~nm}$ reached 0.5 . A $10 \%$ sodium dodecyl sulfate-polyacrylamide gel electrophoresis (SDS-PAGE) was performed to analyze the fusion protein.

\subsection{Identification of BmSirt2}

The band corresponding to our recombinant BmSirt2 was excised as circular plugs 2-3 mm in diameter, and transferred to $1.5 \mathrm{ml}$ Eppendorf tubes. Coomassie blue-stained gel pieces were first destained with $50 \mathrm{ul} \mathrm{of} 50 \% 50 \mathrm{mM} \mathrm{NH}_{4} \mathrm{HCO}_{3}$ and $50 \%$ acetonitrile for $10 \mathrm{~min}$, followed by three washes with $50 \mathrm{ul}$ of MilliQ water. The gel pieces were then dehydrated with 100\% acetonitrile for $5 \mathrm{~min}$, and dried in a SpeedVac (Thermo Sacant) for 30 minutes. The dried gel particles were rehydrated at $4^{\circ} \mathrm{C}$ for $30 \mathrm{~min}$ with $2.5 \mathrm{uL} /$ well trypsin (sequencing grade; Promega, Madison, WI) in 50 $\mathrm{mM} \mathrm{NH}_{4} \mathrm{HCO}_{3}(20 \mathrm{ug} / \mathrm{mL})$, and then incubated at $37^{\circ} \mathrm{C}$ overnight. Peptide mixture $(1 \mathrm{ul})$ was mixed with $1 \mathrm{ul} 10 \mathrm{mg} / \mathrm{ml}$ a-Cyano-4-hydroxycinnamic acid (Sigma) and pointed on the MTP Anchor Chip (Bruker) and analyzed by matrix-assisted laser desorption/ionization-time of flight mass spectrometry (MALDI-TOF-MS) (ultraflex tof/tof, Bruker, Germany). The mass spectra were interpreted using the Mascot peptide mass fingerprint engine available on the web site (http://www.matrixscience.com) for protein identification. To identify the fusion protein further, the western blotting was performed using anti-His-tag antibody (TIANGEN, Beijing).

\section{Results}

\subsection{The analysis of the nucleotide and amino acid sequences}

The sequence of a $1586 \mathrm{bp} \mathrm{B.} \mathrm{mori} \mathrm{cDNA} \mathrm{was} \mathrm{obtained} \mathrm{from} \mathrm{GenBank} \mathrm{under} \mathrm{accession} \mathrm{no.} \mathrm{AB194684,} \mathrm{which} \mathrm{contains}$ an ORF of 1164 bp encoding 387 amino acid residues sequence with a predicted molecular mass and isoelectric point of $43.37 \mathrm{kDa}$ and 5.02, respectively (Figure 1). The amino acid sequence had a conserved SIR2 domain, which contains 183 amino acid residues from the position 334 to 882. No TATA box was found but two poly-A signals existed, which showed as AATAAA (Figure 1). Blasting the cDNA to contigs of B. mori genome in GenBank revealed that contig19687, Ctg001400, contig176714, contig484962 and contig544692 having a high similarity. After analyzing these five contigs, we found that contig19687 contained Ctg001400, contig176714, contig484962 and contig544692. Using SIM4, seven exons were found in the relevant DNA sequence. The length of the exons was 34bp, 267bp, 207bp, 150bp, $193 \mathrm{bp}, 187 \mathrm{bp}$ and $126 \mathrm{bp}$, respectively (Figure 2). Furthermore, we also found BmSirt2 gene was located on Chromosome 25.

\subsection{Protein prediction and analysis}

The deduced amino acid sequence shared $62 \%$ identity to Apis mellifera, 61\% identity to Tribolium castaneum and Nasonia vitripennis, 60\% identity to Anopheles gambiae, 59\% identity to Ciona intestinalis and Homo sapiens, and $56 \%$ identity to Mus musculus. Multiple sequence alignment (Figure 3) showed that the deduced amino acid sequence of Sirt2 from B. mori shared high level identity among these sequences in the conserved domain, SIR2 Domain. To investigate the evolutionary relationships between Sirt2 of B. mori and other organisms, phylogenetic analysis was performed by neighbour-joining (NJ) method using MEGA 3.1 software (Kumar, Tamura, \& Nei, 2004, pp.150-161). Phylogenetic relationship analysis was showed in Figure 4.

\subsection{Cloning, sequencing and gene distribution}

The DNA fragment was obtained through PCR (Figure 5A) and the expression plasmid pET-30a/Sirt2 was successfully constructed. (Figure 5B). The result of sequencing showed that there were two different nucleotide acids, which was pointed in Figure 1. But this mutation was a kind of nonsense mutation and their amino acid residuces didn't change. 
Through semi-quantitative RT-PCR, BmSirt2 gene was detected in multiple tissues, including epidermis, midgut, fat body, silk gland, hemolymph, nerve knot, testis and ovary. This gene was expressed in all these tissues (Figure 6), which suggested that BmSirt2 was a very important and widely expressed gene in B. mori.

\subsection{Expression of BmSirt2}

BmSirt2 was successfully expressed in transformed E. coli BL21 with the pET-30a vector. After induction with IPTG over night at $28^{\circ} \mathrm{C}$, the $E$. coli cells were collected from LB medium. SDS-PAGE analysis revealed that the recombination protein was overexpressed in a soluble form. Since the fusion protein had a His-tag at the N-terminus, its molecular weight was $48.0 \mathrm{kDa}$ (Figure 7) which was higher than that of the predicted mass of $43.37 \mathrm{kDa}$.

\subsection{Identification of BmSirt2}

Two methods were utilized to identify the recombination protein. After identification by MALDI-TOF-MS, the peptide fragments of the recombination protein matched with the deduced amino acid sequence of Sirt2 from B. mori was showed in Figure 8 and Table 1. It indicated that the recombination protein we got was proved to be the BmSirt2 protein. Simultaneity, the western blotting using anti-His-tag antibody revealed that the fusion protein was the one we wanted to get.

\section{Discussion}

Silkworm (B. mori) is an important economic insect and is regarded as a model insect of Lepidoptera. Studies on structures and functions of certain related genes in B. mori have attracted more and more attention.

In this study, we cloned and analyzed the nucleotide sequences of BmSirt2 gene and further predicted its conserved domain. The gene includes an ORF of 1164 bp encoding 387 amino acid residues and the ORF contains seven exons. BmSirt2 gene locates on Chromosome 25 and is showed as a single copy gene. The deduced amino acid sequences show high identity to many other organisms, including from protista to mammals, especially among the SIR2 domain, which indicated that Sirt2 gene is conserved from invertebrates to mammals. Phylogenetic relationship analysis showed that the BmSirt2 protein was in the same subgroup as the Sirt2 from invertebrate animals, such as Tribolium castaneum, Apis Mellifera, Aedes aegypti, Anopheles gambiae, and Drosophila melanogaster.

BmSirt2 gene was widely expressed in B. mori, including epidermis, midgut, fat body, silk gland, hemolymph, nerve knot, testis and ovary. It indicated that BmSirt2 gene may play an important role in many biological processes in almost all the tissues in B. mori. BmSirt2 was successfully expressed and identified by MALDI-TOF-MS and western blotting. The results showed that the recombination protein we got was proved to be the BmSirt2 protein. BmSirt2 can be expressed in a soluble form in E.coli and its fusion protein can be purified using the Ni resin. Thus, further study like enzyme activity assay could be operated to indicate the function of BmSirt2.

BmSirt2 gene is a kind of silence information regulator, which also has the $\mathrm{NAD}^{+}$-dependent deacetylase activity. Recently, many researches found that Sirt2 is a strong candidate to regulate calorie restriction (CR) in many model organisms, such as yeast, nematodes, fruitflies and even mammals (Guarente, 2005, p.923). Guarente and Picard (2005, pp.473-480) reported that limiting calories resulted in activation of the Sir2-family of protein deacetylases, and that this activation accounted for the reduced rate of aging and increased life span observed in response to CR (Masoro, 2004, pp.591-594; Guarente \& Picard, 2005, pp.473-480). Therefore, we used silkworms to study BmSirt2 gene. We thought it was so significant that it would be used to explain the mechanism of longevity which is the eternal topic in life science.

\section{References}

Avalos, J. L., Bever, K. M. \& Wolberger, C. (2005). Mechanism of sirtuin inhibition by nicotinamide: altering the $\mathrm{NAD}(+)$ cosubstrate specificity of a Sir2 enzyme. Molecular Cell, 17, 855-868.

Chakrabarty, S. P., Saikumari, Y. K., Bopanna, M. P. \& Balaram, H. (2008). Biochemical characterization of Plasmodium falciparum Sir2, a NAD+-dependent deacetylase. Molecular Biochemical Parasitology, 158, 139-151.

Frye, R. A. (2000). Phylogenetic classification of prokaryotic and eukaryotic Sir2-like proteins. Biochemical and biophysical research communications, 273, 793-798.

Guarente, L. (2005). Calorie restriction and SIR2 genes--towards a mechanism. Mechanisms of Ageing and Development, 126, 923-928.

Guarente, L. \& Picard, F. (2005). Calorie restriction--the SIR2 connection. Cell, 120, 473-482.

Hoff, K. G., Avalos, J. L., Sens, K. \& Wolberger, C. (2006). Insights into the Sirtuin Mechanism from Ternary Complexes Containing NAD+ and Acetylated Peptide. Structure, 14, 1231-1240.

Kumar, S., Tamura, K. \& Nei, M. (2004). MEGA3: Integrated software for Molecular Evolutionary Genetics Analysis and sequence alignment. Brief Bioinform, 5, 150-163.

Landry, J. \& Sternglanz, R. (2003). Enzymatic assays for NAD-dependent deacetylase activities. Methods, 31, 33-39. 
Masoro, E.J. (2004). Role of sirtuin proteins in life extension by caloric restriction. Mechanisms of Ageing and Development, 125, 591-594.

North, B. J. \& Verdin, E. (2004). Sirtuins: Sir2-related NAD-dependent protein deacetylases. Genome Biology, 5, 224.

Schuetz, A., Min, J., Antoshenko, T., Wang, C.L., Allali-Hassani, A., Dong, A., Loppnau, P., Vedadi, M., Bochkarev, A., Sternglanz, R. \& Plotnikov, A.N. (2007). Structural basis of inhibition of the human NAD+-dependent deacetylase SIRT5 by suramin. Structure, 15, 377-389.

Wang, Q., Chen, K., Yao, Q., Zhao, Y., Li, Y., Shen, H. \& Mu, R. (2008). Identification and characterization of a novel 1-Cys peroxiredoxin from silkworm, Bombyx mori. Comparative Biochemistry and Physiology Part B: Biochemistry Molecular Biology, 149, 176-182.

Table 1. Results of NCBInr Database Search for the Identification of BmSirt2

\begin{tabular}{|c|c|c|c|c|c|}
\hline $\begin{array}{l}\text { Start - } \\
\text { End }\end{array}$ & Observed & $\operatorname{Mr}(\operatorname{expt})$ & $\operatorname{Mr}($ calc $)$ & Delta & Sequence \\
\hline $34-45$ & 1494.8590 & 1493.8517 & 1493.6933 & 0.1585 & R.NMFRDLDVDDVR.M \\
\hline $38-45$ & 946.4945 & 945.4872 & 945.4404 & 0.0469 & R.DLDVDDVR.M \\
\hline $52-68$ & 1867.1019 & 1866.0947 & 1865.9411 & 0.1536 & K.LGLFSPQDLEPAEPPEK.V \\
\hline $89-108$ & 2004.2975 & 2003.2902 & 2003.1051 & 0.1851 & K.KIITLSGAGISTSAGIPDFR.S \\
\hline $90-108$ & 1876.19 & 1875.1884 & 1875.0102 & 0.1782 & K.IITLSGAGISTSAGIPDFR.S \\
\hline $109-120$ & 1386.8173 & 1385.8100 & 1385.6939 & 0.1161 & R.SPETGLYHNLQK.Y \\
\hline $121-136$ & 2012.2227 & 2011.2154 & 2011.0203 & 0.1951 & K.YELPQPQAIFEINFFR.Q \\
\hline $137-147$ & 1273.8038 & 1272.7966 & 1272.6867 & 0.1099 & R.QNPKPFFTLAK.E Gln->pyro-Glu (N-term Q) \\
\hline $137-147$ & 1290.8409 & 1289.8337 & 1289.7132 & 0.1205 & R.QNPKPFFTLAK.E \\
\hline $148-164$ & 2039.2980 & 2038.2907 & 2038.0676 & 0.2231 & K.ELFPGSFKPTISHYFIR.L \\
\hline $175-185$ & 1389.8080 & 1388.8007 & 1388.6684 & 0.1323 & R.HYTQNIDTLER.G \\
\hline $194-210$ & 2066.1602 & 2065.1529 & 2064.9146 & 0.2383 & .LVEAHGTFYTSHCLDCR.K \\
\hline $194-211$ & 2194.2659 & 2193.2586 & 2193.0096 & 0.2490 & K.LVEAHGTFYTSHCLDCRK.E \\
\hline $211-219$ & 1152.7128 & 1151.7055 & 1151.6226 & 0.0829 & R.KEYPLEFVK.E \\
\hline $212-219$ & 1024.6399 & 1023.6326 & 1023.5277 & 0.1049 & K.EYPLEFVK.E \\
\hline $212-221$ & 1309.8035 & 1308.7962 & 1308.6714 & 0.1248 & K.EYPLEFVKER.I \\
\hline $297-307$ & 1235.8621 & 1234.8548 & 1234.7510 & 0.1038 & R.EKAGVRPPILR.I \\
\hline $299-307$ & 978.7280 & 977.7207 & 977.6134 & 0.1073 & K.AGVRPPILR.I \\
\hline $310-324$ & 1655.9109 & 1654.9036 & 1654.7443 & 0.1593 & R.GLMCGGLQLDEGSYR.D \\
\hline $310-328$ & 2097.2471 & 2096.2398 & 2095.9779 & 0.2619 & R.GLMCGGLQLDEGSYRDVAR.L \\
\hline $329-342$ & 1623.7961 & 1622.7889 & 1622.6301 & 0.1588 & R.LGDCDEGCQDLADR.L \\
\hline $343-350$ & 945.5690 & 944.5618 & 944.4716 & 0.0902 & R.LGWGDELR.A \\
\hline
\end{tabular}

There listed the start and end position of the matched peptide, the observed molecular mass, expective Mr, calculative $\mathrm{Mr}$, the delta value between expective $\mathrm{Mr}$ and calculative $\mathrm{Mr}$ and the matched sequences. 
1

61

121

181

241

301

361

481

541

601

661

721

781

841

901

961

1021

1081

1141

1201

1261 TCGGATGACCTGAATGAAGOGAAGAAAAGCCATGATGTGATTCAAATAAAAATAT TGGAA 1321 AATT AATAAAAAT T TACAGATATGTAAATCGAACTACAAGCACCT TCT TGT TCTAT T TAA 1381 TAAT AATT TAAAAAGGTACTTAG TAAG AAAT ACTCAAT T TAATAT TCGCTCTATT T TCAT 1441 GCGGT TAATACAGATAAAATTCTCTCTGGG TCTCCGGT T TCTAT TOGAT TTCAAAGAAG T 1501 ACGCAAACAATATACTCATAAAT TT TTG TATATAGCAACATTCAATTAT TTAT TGGTAAA 1561 CTGAAAAOCACTTCAAG TAOCTAAT T

Figure 1. The cDNA sequence and deduced amino acid sequence of BmSirt2. The start codon (ATG) and stop codon (TGA) are boxed. Two poly-A signals are underlined. In amino acid sequences, SIR2 domain is framed. Two nonsense mutation found in the sequencing are shaded. 


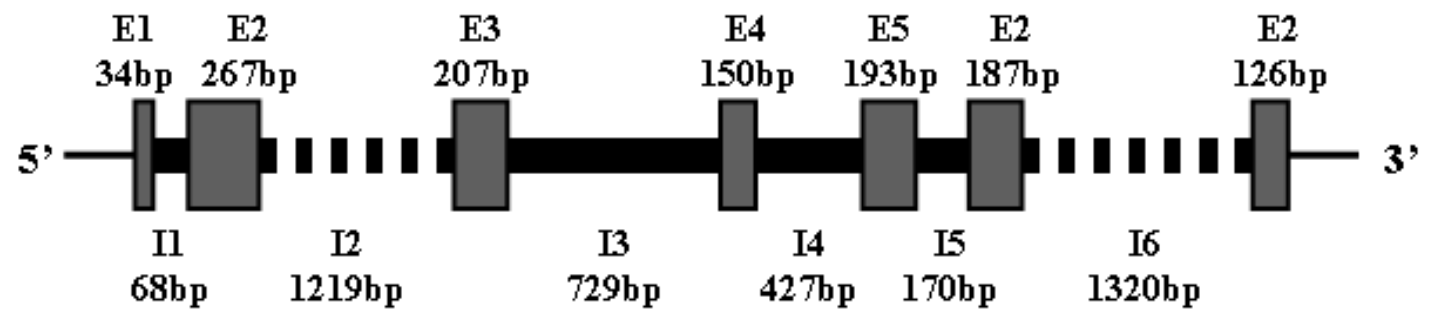

Figure 2. The gene structure of BmSirt2. Seven exons (E) are indicated as large open boxes with the length in nucleotides just above the box. Introns (I) are thick lines with lengths in nucleotides listed blow the lines.
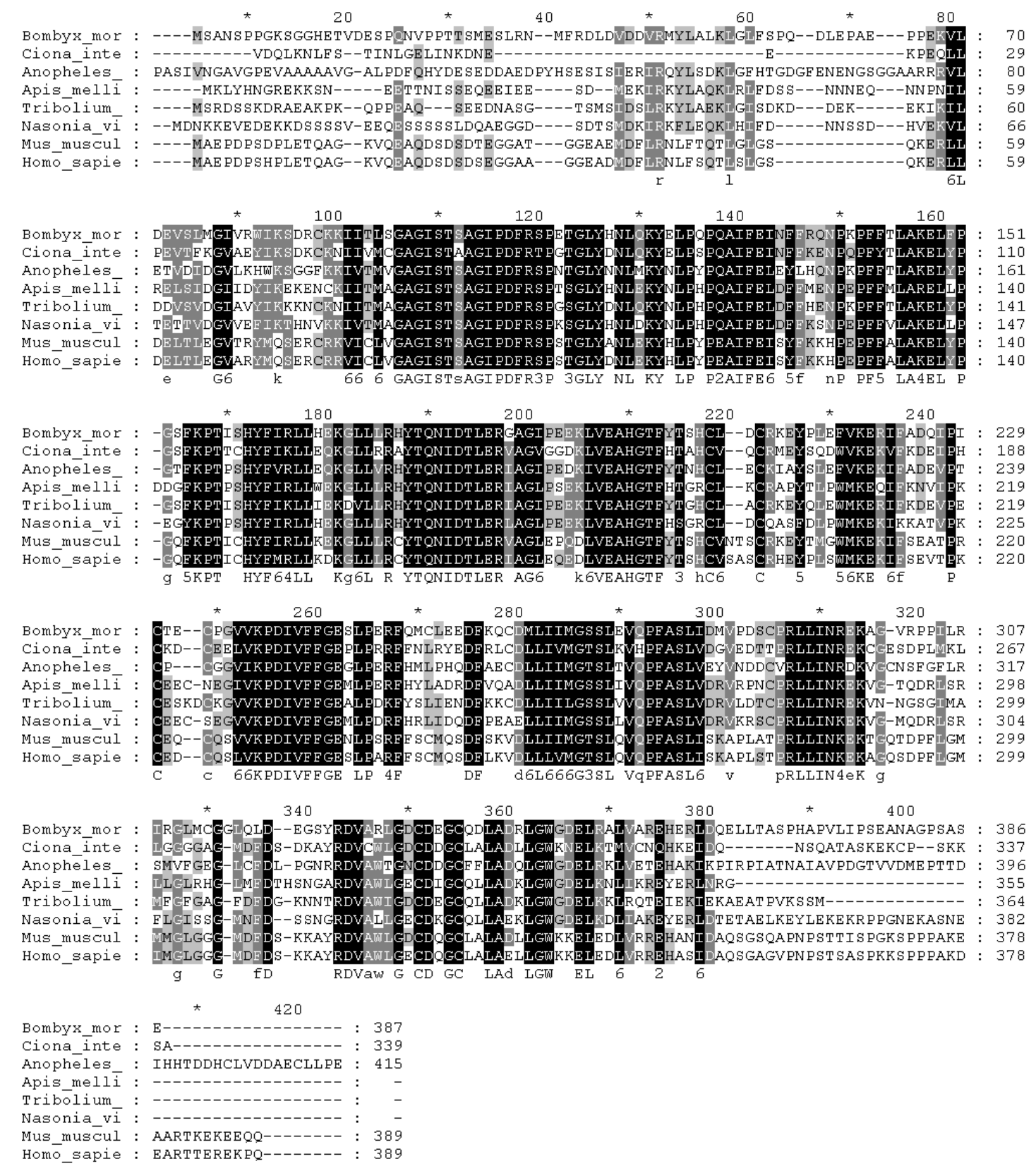

Figure 3. Multiple alignments of the amino acid sequences of Sirt2 proteins from B. mori, Ciona intestinalis, Anopheles gambiae, Apis mellifera, Tribolium castaneum, Nasonia vitripennis, Mus musculus and Homo sapiens. Invariant residues are highlighted in black. 


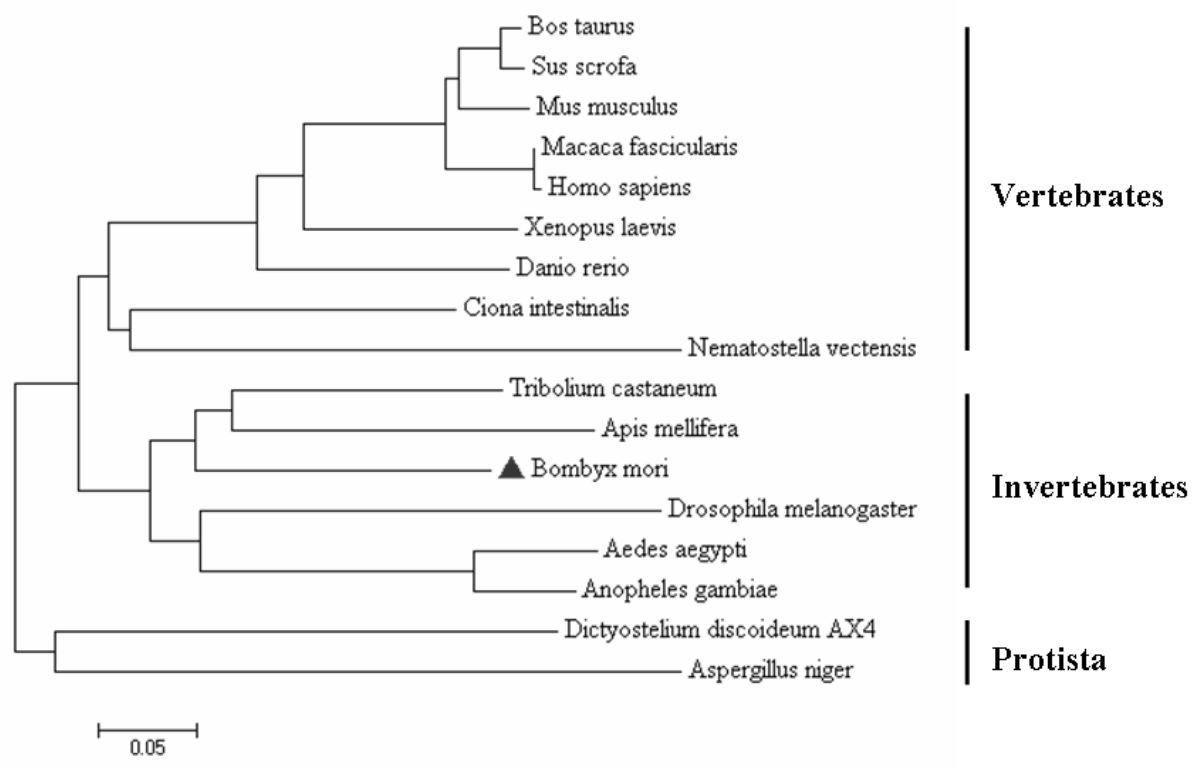

Figure 4. Neighbour-joining phylogram showing the relationships between Sirt2 from B. mori and other organisms. The tree distances were generated according to the ClustalW algorithm, and the tree was constructed using MEGA 3.1.

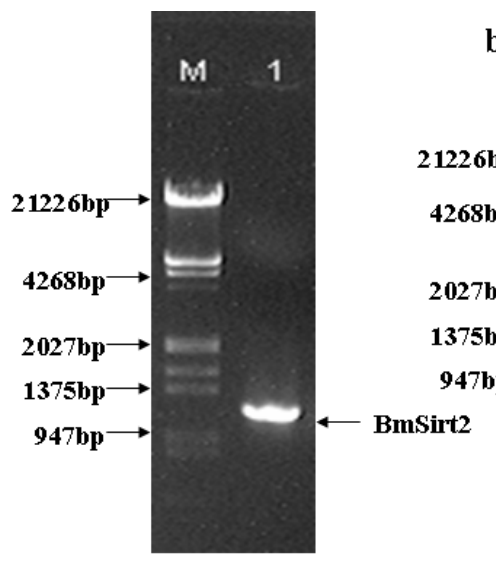

A

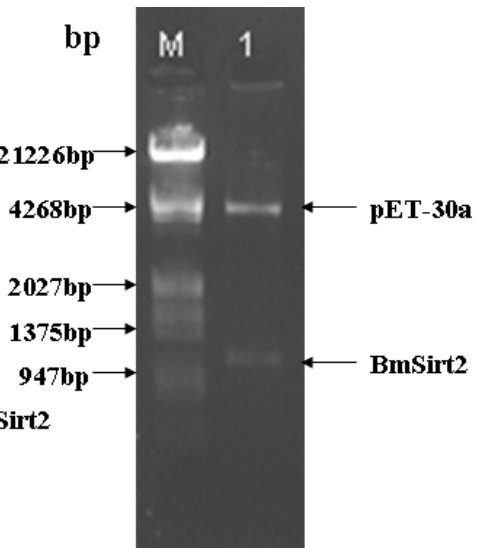

B

Figure 5. (A) The result of PCR for BmSirt2. Lane M, DNA molecular mass maker; Lane 1, PCR product. (B) Identification of the recombinant plasmid pET-30a/BmSirt2 by digestion by BamHI and XhoI. Lane M, DNA molecular mass maker; Lane 1, digested pET-30a/BmSirt2

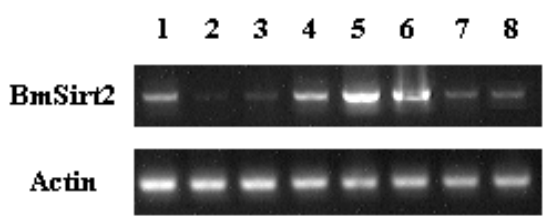

Figure 6. Distribution of BmSirt2 gene in multiple tissues. 1. epidermis; 2. midgut; 3. fat body; 4. silk gland; 5. hemolymph; 6. nerve knot; 7. testis; 8. ovary. B. mori Actin A3 gene was used as the control to show the normalization of the amount of templates in PCR reactions. 


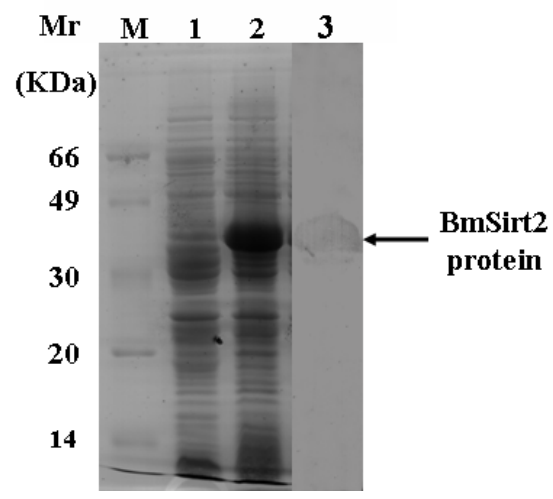

Figure 7. Expression of BmSirt2 in E. coli. BL21 cells. Cell extracts were separated by SDS-PAGE and stained with Coomassie brilliant blue. Lane M: protein molecular weight markers. Lane 1: proteins from BL21 transformed with pET-30a plasmid. Lanes 2: proteins from BL21 cells transformed with plasmid containing BmSirt2 gene. Lane 3: the western blotting of fusion protein using anti-His-tag antibody.

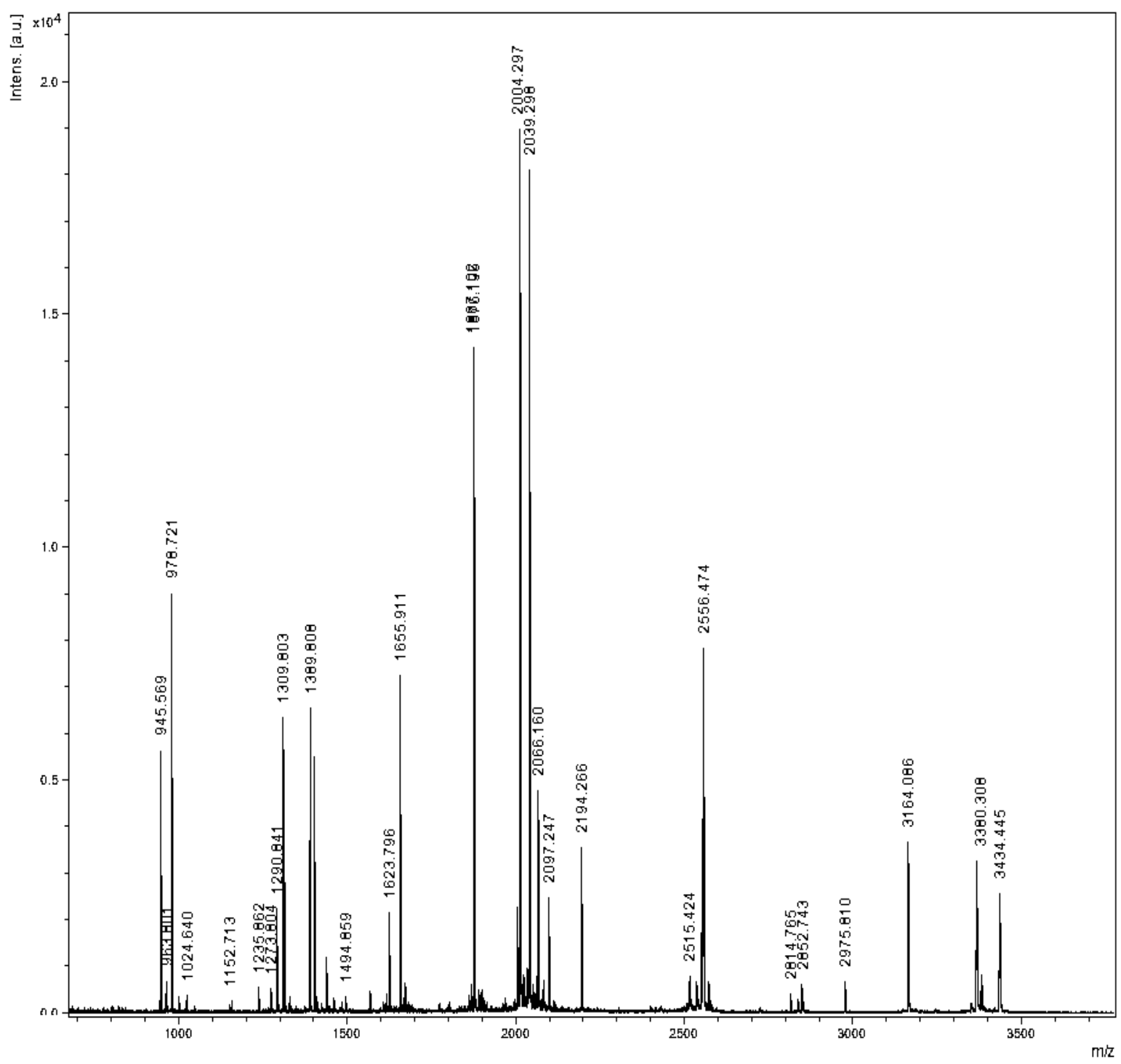

Figure 8. Peptide mass fingerprints (PMF) for BmSirt2. MALDI-TOF-MS spectrometry was done with in-gel-digested peptides of the recombination protein. 